\title{
Influence of an intratumoral cyst on drug distribution by convection-enhanced delivery: case report
}

\author{
Iryna Ivasyk, BS, ${ }^{1}$ Peter F. Morgenstern, MD, ${ }^{1,2}$ Eva Wembacher-Schroeder, BS, ${ }^{3}$ and \\ Mark M. Souweidane, MD ${ }^{1,2}$
}

1Department of Neurological Surgery, NewYork-Presbyterian Hospital, Weill Cornell Medicine; '2Department of Neurosurgery, Memorial Sloan Kettering Cancer Center, New York, New York; and ${ }^{3}$ Brainlab, Munich, Germany

\begin{abstract}
Convection-enhanced delivery (CED) uses positive pressure to induce convective flow of molecules and maximize drug distribution. Concerns have been raised about the effect of cystic structures on uniform drug distribution with CED. The authors describe the case of a patient with a diffuse intrinsic pontine glioma (DIPG) with a large cyst and examine its effect on drug distribution after CED with a radiolabeled antibody. The patient was treated according to protocol with CED of ${ }^{124} \mathrm{I}-8 \mathrm{H} 9$ to the pons for nonprogressive DIPG after radiation therapy as part of a Phase I trial (clinical trial registration no. NCT01502917, clinicaltrials.gov). Care was taken to avoid the cystic cavity in the planned catheter track and target point. Co-infusion with Gd-DTPA was performed to assess drug distribution. Infusate distribution was examined by MRI immediately following infusion and analyzed using iPlan Flow software. Analysis of postinfusion MR images demonstrated convective distribution around the catheter tip and an elongated configuration of drug distribution, consistent with the superoinferior corticospinal fiber orientation in the brainstem. This indicates that the catheter was functioning and a pressure gradient was established. No infusate entry into the cystic region could be identified on T2-weighted FLAIR or T1-weighted images. The effects of ependymal and pial surfaces on drug delivery using CED in brainstem tumors remain controversial. Drug distribution is a critical component of effective application of CED to neurosurgical lesions. This case suggests that cyst cavities may not always behave as fluid "sinks" for drug distribution. The authors observed that infusate was not lost into the cyst cavity, suggesting that lesions with cystic components can be treated by CED without significant alterations to target and infusion planning.
\end{abstract}

https://thejns.org/doi/abs/10.3171/2017.5.PEDS1774

KEY WORDS convection-enhanced delivery; tumor cyst; diffuse intrinsic pontine glioma; DIPG; brainstem tumor; surgical technique; oncology

$\mathrm{C}$

ONVECTION-ENHANCED delivery (CED) enables localized drug delivery to tissues using positive pressure L induce convective flow of molecules and achieve greater distribution of drug than other diffusion-based methods. CED is of particular interest in treating tumors of the CNS as it overcomes the blood-brain barrier (BBB), currently a central limitation of drug delivery for these tumors. The efficacy of CED is limited by factors such as infusion rate, with higher rates reaching a larger volume of distribution but increasing the likelihood of adverse complications. ${ }^{12}$ Additionally, selection of a target and accu- racy of catheter placement factor into CED success, both in regard to drug distribution and mitigating risk of injury to structures that must be traversed..$^{16,18,19}$

Furthermore, the effect of ependymal and pial surfaces on drug delivery through CED in brainstem tumors remains controversial. Specifically, one study identified a drastic decrease in the ratio of infusion volume to the volume of distribution of the drug when the infusion front reached the pial surface. ${ }^{10}$ Others found that leakage into the subarachnoid space might be possible through the ependymal surface, but that the pia provides an absolute bar- 
rier for large molecules unless it is perforated by the catheter. ${ }^{18}$ Furthermore, it has been suggested that selecting targets away from cystic structures can be beneficial by preventing loss of infusate into the cyst cavity. ${ }^{20}$ Here, we present a case of a diffuse intrinsic pontine glioma (DIPG) with an intratumoral cyst and discuss the resulting effects on drug distribution achieved using CED.

\section{Case Report}

\section{Clinical History}

This 7-year-old girl initially presented with intermittent morning headaches and gait instability. The patient was referred by her pediatrician to a neurologist, who noted a right cranial nerve VI palsy and right hemiparesis, as well as horizontal nystagmus. The patient underwent MRI of the brain, which demonstrated an infiltrative expansile mass centered in the pons, consistent with DIPG. She was started on a regimen of dexamethasone and treated with conventional radiation therapy (59.4 Gy).

A 1-month posttreatment MRI study demonstrated reduction in tumor volume as well as a peripherally enhancing cystic area in the left pons. A follow-up MRI study obtained weeks later demonstrated an increase in the size of the cystic lesion, for which a drainage procedure was performed with simultaneous stereotactic tumor biopsy. Histological analysis was consistent with DIPG, and immunostaining was suggestive of the presence of $H 3 K 27 M$ mutation.

Sequential MRI studies demonstrated asymptomatic recurrence of the cyst. After informed consent, the patient was enrolled into the Phase 1 study, "Convection-Enhanced Delivery of 124I-8H9 for Patients with Non-Progressive Diffuse Pontine Gliomas Previously Treated with External Beam Radiation Therapy" (clinical trial registration no. NCT01502917, clinicaltrials.gov). She underwent infusion of $4000 \mathrm{mCi}$ of ${ }^{124} \mathrm{I}-8 \mathrm{H} 9$ at a maximal rate of 5 $\mu \mathrm{l} / \mathrm{min}$ without any adverse events 27 days after the cyst drainage procedure. Given her stable disease status, the patient qualified for and underwent a second treatment with the same infusion parameters 2 months later.

\section{Operation}

The surgical planning procedure for the infusion catheter (Brainlab Flexible Catheter) was performed using iPlan Flow (Brainlab), FDA-approved software specifically designed to support the planning of intracranial drug delivery. MRI-guided stereotactic placement of the catheter was performed after induction of general anesthesia and using the ClearPoint system (MRI Interventions, Inc.), an MRI-compatible skull-mounted guidance device, which has previously been described in detail. ${ }^{15}$

The insertion was performed in the BrainSuite (Brainlab), an intraoperative imaging suite that includes a $1.5-\mathrm{T}$ Siemens scanner (Siemens AG). The catheter has a stylet that is removed following placement, allowing distal flexibility. Once the catheter is in place, a bone anchor is used to secure the catheter, and the guidance device can be removed. The infusion of ${ }^{124} \mathrm{I}-8 \mathrm{H} 9$ with co-infusion of Gd-DTPA (0.5 mol/L Magnevist [Bayer Schering Phar$\mathrm{ma}$, diluted in saline to a concentration of $5 \mathrm{mmol} / \mathrm{L}$ ), was administered via CED outside the MR environment in a monitored setting at a maximal rate of $5 \mu \mathrm{l} / \mathrm{min}$. The patient experienced no acute toxicity or neurological complications.

\section{Imaging Analysis}

To assess agent distribution, MRI (high-resolution T1-weighted imaging with and without intravenous contrast, T2-weighted imaging, diffusion tensor imaging, and FLAIR imaging, slices $\leq 3 \mathrm{~mm}$ ) was performed as soon as possible after completion of infusion ( $<2$ hours). For analysis of the Gd-DTPA infusion, the postinfusion MR images were imported into the iPlan Flow planning software and merged with the pre- and intraoperative images using the automatic imaging fusion functionality of the software.

T2-weighted, T1-weighted, and FLAIR images were the basis to identify the volume of the cystic component and the target region in iPlan Flow (Fig. 1). The actual infusate distribution was analyzed on the high-resolution T1-weighted MR and FLAIR images. The volume of distribution was segmented by applying a custom-made semiautomatic segmentation algorithm involving background subtraction and normalization.

Analysis of the MR images obtained in this patient reveals convective distribution in a radial arrangement around the catheter tip and with an ellipsoid configuration that is consistent with the longitudinal fiber tracts through the brainstem (Fig. 2). Importantly, no surrogate tracer was detected within the intratumoral cyst (Fig. 3). ${ }^{10}$

\section{Discussion}

In the pediatric population, brainstem tumors account for $10 \%-20 \%$ of all CNS cancers. These tumors are further subdivided into low-grade focal brainstem gliomas and DIPGs. ${ }^{8}$ DIPGs, which account for $80 \%$ of all brainstem tumors, typically arise in 7- to 9-yearold children, with no preference regarding sex. These tumors typically present as a progressive accumulation of symptoms characteristic of cerebellar dysfunction and cranial nerve palsies (most frequently cranial nerves VI and VII), such as ataxia, facial weakness, diplopia, and hearing loss. ${ }^{3,6,11,21,22}$

A diagnosis of DIPG is traditionally made based on clinical presentation and MRI findings. Palliative radiation therapy is currently the standard of care. Because of its diffuse infiltrative nature and eloquent location, a DIPG is not amenable to resection. Clinical trials of various chemotherapeutic agents with and without radiation have failed to show a benefit. The mean overall survival of patients with DIPG is estimated at 9-14 months after diagnosis, and even the most aggressive radiotherapy regimen can prolong this time only by $3-6$ months. ${ }^{21}$

The relative impermeability of the BBB to systemically administered chemotherapeutic agents presents a challenge in the treatment of CNS malignancies. Strategies to overcome this hurdle and deliver local therapy, such as BBB disruption with mannitol or focused ultrasound, have shown some promise. ${ }^{4,21}$ Preliminary studies have suggested that CED is a safe and effective method of overcoming the $\mathrm{BBB}$ and achieving high local concentrations 

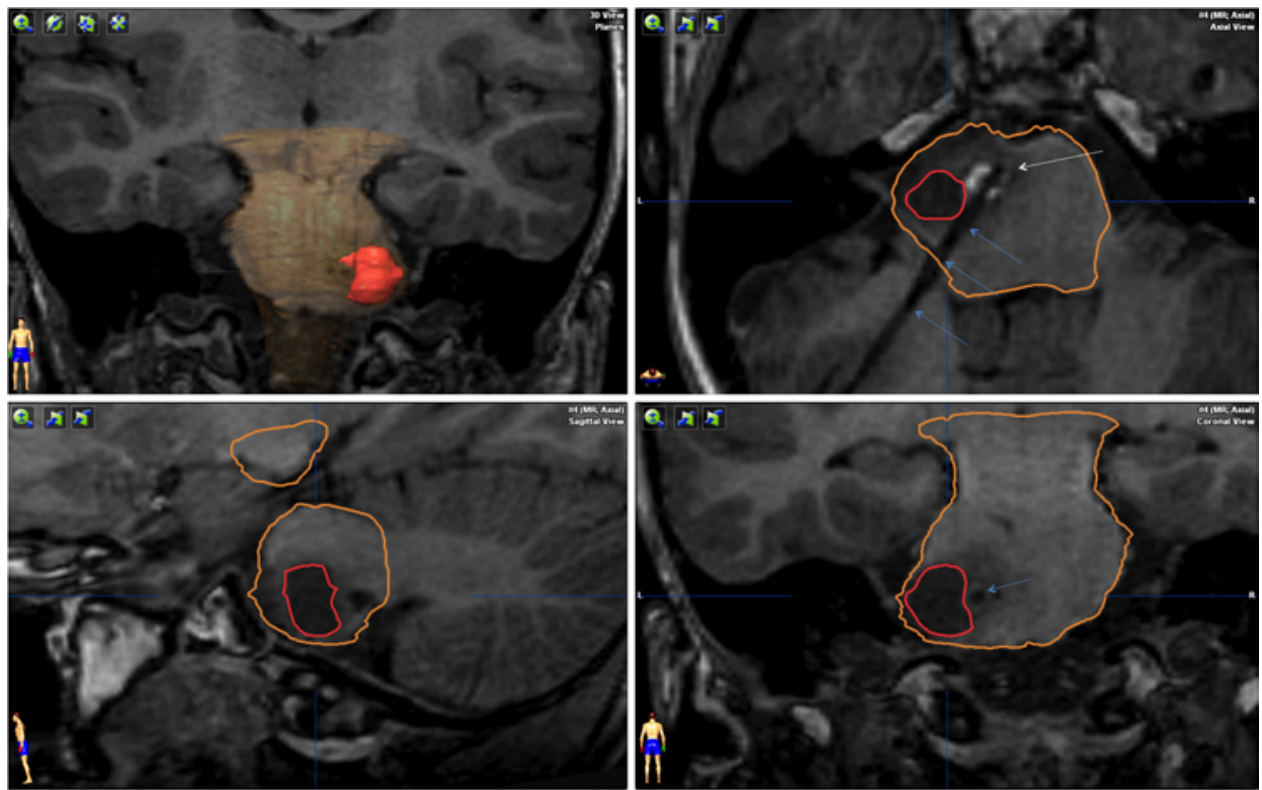

FIG. 1. Reconstructed view of 3D T1-weighted MR images without Gd showing a cystic lesion (red outline) and segmented brainstem (orange outline). The ventricular catheter tract is visible as a signal void (blue arrows). Hyperintensity around the catheter tip corresponds with blood products (white arrow).

of the drug. CED uses positive pressure to overcome the limitations of diffusion and simulate bulk molecular flow, which can be monitored in real time with a co-infusion of imaging tracers. ${ }^{2,5,7,9,12,13}$ Limitations of CED include factors such as a balance of infusion rate that reaches a reasonable area of distribution but does not lead to edema or neurological injury, as well as the complexity associated with precise placement of the catheter in a deep eloquent location..$^{12,18}$

Multiple studies have examined the effects of pial and ependymal surfaces on drug distribution using CED. Using injections of both high-molecular-weight (HMW) and low-molecular-weight compounds into the brains of nonhuman primates, Jagannathan et al. demonstrated leakage into the subarachnoid space through pial and ependymal surfaces regardless of particle size.$^{10}$ However, Sampson et al. found that pial surfaces can prevent HMW compounds from entering the subarachnoid space, provided that the pial surface is not punctured during catheter placement. ${ }^{18}$
The effect of drug "leaking" into the CSF presents a limitation for this method of drug delivery that should be considered as a factor in treating tumors located close to this boundary. In the case presented, the shortest distance from the catheter tip to the cavity wall was more than 10 $\mathrm{mm}$, which is similar to the previously reported recommended minimum distance from fluid-filled structures for CED. ${ }^{14,17}$ Based on such studies, it has been postulated that cystic lesions within the target region might behave similarly to the subarachnoid space and serve as a low-pressure conduit into which infusate might preferentially pool, creating a barrier in volume and concentration of drug that can be delivered. In the case presented, we observed convective distribution around the catheter tip over a clinically relevant volume of the brainstem. However, despite prevailing theory, there was no evidence of infusate leakage into the tumor cyst.

It is well known that the coadministration of tracers to estimate drug distribution is a useful strategy but has
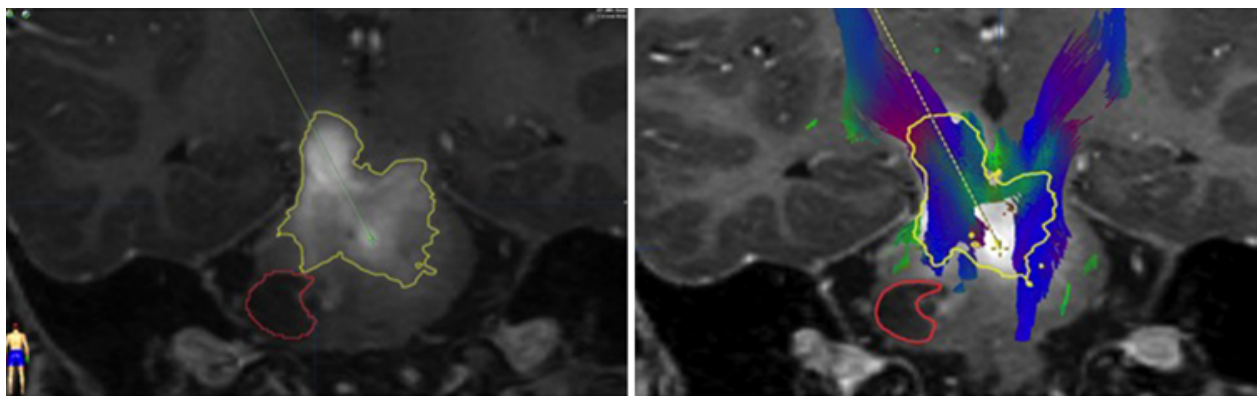

FIG. 2. Left: Coronal 3D T1-weighted MR image with intravenous contrast demonstrates the cystic lesion (red outline) and GdDTPA distribution (yellow outline) around the catheter tip location (green crosshair). Right: The configuration of drug distribution is consistent with the superoinferior corticospinal fiber orientation in the brainstem. 

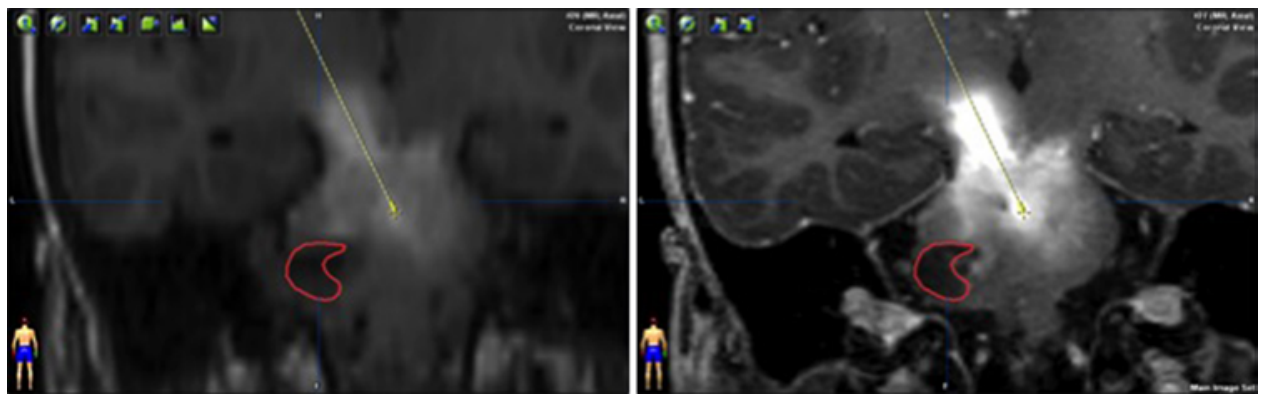

FIG. 3. Coronal FLAIR image (left) and 3D T1-weighted MR image without intravenous contrast (right) demonstrate close approximation of the Gd-DTPA distribution to the cyst wall without entry into the cyst itself. This MR image was acquired immediately after completion of the Gd-DTPA infusion.

its own limitations. For example, Gd-DTPA is a smaller molecule than the therapeutic infusate in this case ${ }^{124} \mathrm{I}-$ $8 \mathrm{H} 9)$, a radiolabeled antibody, and therefore may overestimate drug distribution. Our group and others are currently studying alternative strategies to overcome this limitation and achieve more accurate monitoring of infusate distribution at the target.

Our experience in this case suggests that cystic lesions may not adversely affect CED drug distribution and therefore can be treated successfully with this strategy. More thorough investigation is needed to precisely define circumstances in which cystic lesions do not impact drug delivery and distribution and may allow for definition of minimum distance guidelines for CED targeting in relation to cystic structures. The intracystic pressures may differ from those pressures in the subarachnoid space or ventricular compartments. ${ }^{1}$ Further evaluation of drug distribution in a larger series will help better define these phenomena.

One limitation of this case report is that the nature of the cyst and its permeability could be patient-specific, and this outcome would not be widely generalizable to this population. However, further investigation in a larger series with additional preclinical experimental evaluations of drug distribution is needed to validate the hypotheses generated by this case report. This knowledge may aid in identifying patients who are candidates for CED as well as specific anatomical anomalies that may hinder successful treatment.

\section{Acknowledgments}

Ms. Ivasyk was supported by a Medical Scientist Training Program grant from the National Institute of General Medical Sciences of the National Institutes of Health under award no. T32GM007739 to the Weill Cornell/Rockefeller/Sloan Kettering Tri-Institutional MD-PhD Program. This study is solely the responsibility of the authors and does not necessarily represent the official views of the National Institutes of Health.

\section{References}

1. Ali MJ, Navalitloha Y, Vavra MW, Kang EW, Itskovich AC, Molnar P, et al: Isolation of drug delivery from drug effect: problems of optimizing drug delivery parameters. Neuro Oncol 8:109-118, 2006

2. Barua NU, Lowis SP, Woolley M, O'Sullivan S, Harrison R, Gill SS: Robot-guided convection-enhanced delivery of carboplatin for advanced brainstem glioma. Acta Neurochir (Wien) 155:1459-1465, 2013

3. Berger MS, Edwards MS, LaMasters D, Davis RL, Wilson CB: Pediatric brain stem tumors: radiographic, pathological, and clinical correlations. Neurosurgery 12:298-302, 1983

4. Castel D, Philippe C, Calmon R, Le Dret L, Truffaux N, Boddaert N, et al: Histone H3F3A and HIST1H3B K27M mutations define two subgroups of diffuse intrinsic pontine gliomas with different prognosis and phenotypes. Acta Neuropathol 130:815-827, 2015

5. Chittiboina P, Heiss JD, Lonser RR: Accuracy of direct magnetic resonance imaging-guided placement of drug infusion cannulae. J Neurosurg 122:1173-1179, 2015

6. Epstein F, Wisoff JH: Intrinsic brainstem tumors in childhood: surgical indications. J Neurooncol 6:309-317, 1988

7. Goodwin CR, Xu R, Iyer R, Sankey EW, Liu A, Abu-Bonsrah N, et al: Local delivery methods of therapeutic agents in the treatment of diffuse intrinsic brainstem gliomas. Clin Neurol Neurosurg 142:120-127, 2016

8. Green AL, Kieran MW: Pediatric brainstem gliomas: new understanding leads to potential new treatments for two very different tumors. Curr Oncol Rep 17:436, 2015

9. Groothuis DR: The blood-brain and blood-tumor barriers: a review of strategies for increasing drug delivery. Neuro Oncol 2:45-59, 2000

10. Jagannathan J, Walbridge S, Butman JA, Oldfield EH, Lonser RR: Effect of ependymal and pial surfaces on convectionenhanced delivery. J Neurosurg 109:547-552, 2008

11. Littman P, Jarrett P, Bilaniuk LT, Rorke LB, Zimmerman RA, Bruce DA, et al: Pediatric brain stem gliomas. Cancer 45:2787-2792, 1980

12. Lonser RR, Sarntinoranont M, Morrison PF, Oldfield EH: Convection-enhanced delivery to the central nervous system. J Neurosurg 122:697-706, 2015

13. Lonser RR, Schiffman R, Robison RA, Butman JA, Quezado $\mathrm{Z}$, Walker ML, et al: Image-guided, direct convective delivery of glucocerebrosidase for neuronopathic Gaucher disease. Neurology 68:254-261, 2007

14. Patel SJ, Shapiro WR, Laske DW, Jensen RL, Asher AL, Wessels BW, et al: Safety and feasibility of convectionenhanced delivery of Cotara for the treatment of malignant glioma: initial experience in 51 patients. Neurosurgery 56:1243-1253, 2005

15. Richardson RM, Kells AP, Martin AJ, Larson PS, Starr PA, Piferi PG, et al: Novel platform for MRI-guided convectionenhanced delivery of therapeutics: preclinical validation in nonhuman primate brain. Stereotact Funct Neurosurg 89:141-151, 2011

16. Rosenbluth KH, Eschermann JF, Mittermeyer G, Thomson R, Mittermeyer S, Bankiewicz KS: Analysis of a simulation algorithm for direct brain drug delivery. Neuroimage 59:2423-2429, 2012 
17. Sampson JH, Archer G, Pedain C, Wembacher-Schröder E, Westphal M, Kunwar S, et al: Poor drug distribution as a possible explanation for the results of the PRECISE trial. J Neurosurg 113:301-309, 2010

18. Sampson JH, Brady ML, Petry NA, Croteau D, Friedman $\mathrm{AH}$, Friedman HS, et al: Intracerebral infusate distribution by convection-enhanced delivery in humans with malignant gliomas: descriptive effects of target anatomy and catheter positioning. Neurosurgery 60 (2 Suppl 1):ONS89-ONS99, 2007

19. Sampson JH, Raghavan R, Brady ML, Provenzale JM, Herndon JE II, Croteau D, et al: Clinical utility of a patient-specific algorithm for simulating intracerebral drug infusions. Neuro Oncol 9:343-353, 2007

20. van Putten EH, Wembacher-Schröder E, Smits M, Dirven CM: Magnetic resonance imaging-based assessment of gadolinium-conjugated diethylenetriamine penta-acetic acid testinfusion in detecting dysfunction of convection-enhanced delivery catheters. World Neurosurg 89:272-279, 2016

21. Vanan MI, Eisenstat DD: DIPG in children - what can we learn from the past? Front Oncol 5:237, 2015
22. Walker DA, Punt JA, Sokal M: Clinical management of brain stem glioma. Arch Dis Child 80:558-564, 1999

\section{Disclosures}

Ms. Wembacher-Schroeder is an employee of Brainlab.

\section{Author Contributions}

Conception and design: Souweidane. Acquisition of data: Morgenstern, Ivasyk, Wembacher-Schroeder. Analysis and interpretation of data: Morgenstern, Ivasyk, Wembacher-Schroeder. Drafting the article: Morgenstern, Ivasyk, Wembacher-Schroeder. Critically revising the article: all authors. Reviewed submitted version of manuscript: all authors. Approved the final version of the manuscript on behalf of all authors: Morgenstern. Study supervision: Souweidane.

\section{Correspondence}

Peter Morgenstern, Department of Neurological Surgery, NewYorkPresbyterian Hospital, Weill Cornell Medical Center, 525 East 68th St., Box 99, New York, NY 10065. email: pfm2001@nyp.org. 